\title{
Remote Sensing Image Enhancement Algorithm for Water Pollution Monitoring Based on Rational Interpolation
}

\author{
Yang Ning ${ }^{1,2, a}$, Yunfeng Zhang ${ }^{1,2, a}$, Yifang $\mathrm{Liu}^{1,2, a}$, Jun $\mathrm{He}^{1,2, a}$, Caiming \\ Zhang ${ }^{1,3, a}$ \\ ${ }^{1}$ Department of Computer Science and Technology, Shandong University of Finance and \\ Economics, Jinan 250014, China \\ 2 Digital Media Technology Key Lab of Shandong Province, Jinan 250061, China \\ ${ }^{3}$ Department of Computer Science and Technology, Shandong University, Jinan 250100, China \\ apolaris606@163.com
}

Keywords: Remote Sensing Image, Water Pollution Monitoring, Rational Interpolation

\begin{abstract}
Simultaneously increasing the resolution and improving the display quality of the Remote Sensing (RS) imagery, such as water pollution image, is a challenge at present. We propose a new interpolation algorithm based on the rational function model. The model has a free parameter and the shape of the interpolation surface can be modified by using the parameter for the unchanged data. Transformation over triangular domain further improves the interpolation accuracy of the rational function. We divide the image into smooth area and non-smooth area by drawing contour lines and have given full consideration to the directional information of the image edge. Optimization of the parameter makes the interpolation surface more suitable for interpreting the image data directly. The experimental results show that the new techniques not only increase the resolution but also achieve comparative good effects for some RS images.
\end{abstract}

\section{Introduction}

The oil spill in the Gulf of Mexico is one of the largest environmental disasters in recent history. As shown in Fig.1, it has caused serious water pollution (T.J.Crone et al.2010). Nowadays, water pollution monitoring plays an important role in the water management and pollution control. However, relying only on the traditional technology is difficult to meet the requirements of the water pollution detection. Therefore some people try to use the RS image to monitor water pollution. The practices show that analyzing of the RS image might be the best method to deal with the large-scale water pollution.

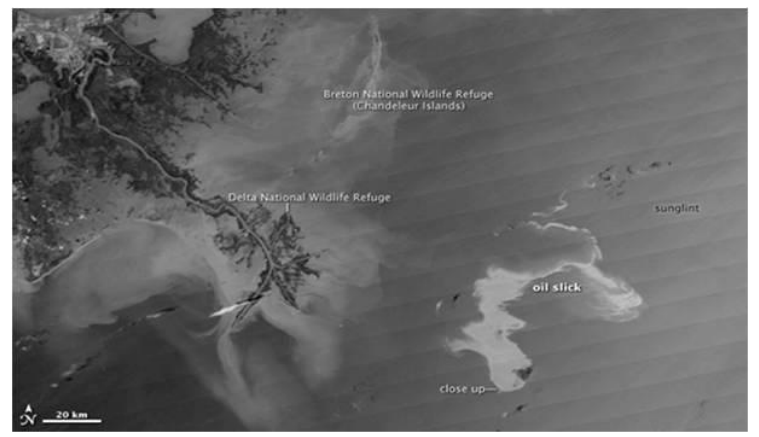

Fig.1 Oil Spill Gulf of Mexico 2010

As a matter of fact, spaceborne synthetic aperture radar (SAR) is well adapted to detect water pollution, especially the ocean pollution, independently from daily or weather conditions (Gregoire Mercier et al. 2006). But the RS images of the SAR systems are sensitive to gravity waves (due to their resolution). In fact, the presence of surface slick, reducing the signal backscattered (Maurizio Migliaccio et al. 2009) to the radar antenna, generates a low-backscattering area

which, in RS images, appears as a dark area (Nunziata

F et al. 2009). Therefore if the RS image resolution is not good enough, it is hard to distinguish the dark area from the look-alike area. Generally, during the process of analyzing the RS image in 
the traditional method, it always reduces the available spatial resolution of the RS image. Sometimes, the spatial extent of the water pollution, such as oil slick, is large enough to be resolvable by the RS image, particularly when processed with resolution enhancement algorithms (Richard D.Lindsley et al 2012).

One important factor for the RS image quality is the speckle. High-resolution data products can have fewer scatterers within each resolution cell, and fully developed speckle may not be obtained. To reduce speckle, both fully and partially developed, multilooking techniques are often applied, at the cost of reduced resolution (Stine Skrunes et al 2015). If it is desired to keep the full resolution and the display quality of the RS image, many advanced interpolation algorithms can be applied.

Over the last decades, various interpolation methods have been proposed. The most widely used traditional interpolation methods are Bi-linear, Bi-cubic, Cubic spline algorithm, etc (R.G Key 1981; H.S Hou et al. 1978). These methods have a relatively low complexity, but the processing of edge is not good enough. Nowadays, many scholars have proposed some algorithms based on wavelets transform (Liu Xiaodi et al. 2003; Liu Bo et al. 2006; Tu Guofang et al. 2005; Qu Youshan et al. 2004; Zhang can et al. 2005). Separable wavelets can capture only limited directional information. Thus, these disappointing behaviors reduce the visual quality of RS images. Although algorithms based on contourlet transform (M.N.Do et al. 2005; M.N.Do et al. 2002; A.L.Cunba et al. 2006; J.Zhou et al. 2005) have nice directional multi-resolution, but due to down-sampling and up-sampling in both the LP and DFB, the contourlet transform is shift-variant. The lack of shift-invariance causes pseudo-Gibbs phenomena around singularities, which is disadvantageous to image interpolation.

In order to solve these problems, many adaptive interpolation algorithms have been proposed. (Li Xin et al. 2001; Zhang Lei et al. 2006; Zhang Xiangjun et al. 2008). All these methods improve the visual effect of RS images. However, these methods interpolate the unknown pixel with a set of sub-pixels which are not suitable for re-sampling after instead of a continuous rational function and sometimes generate speckle noise or distortion of edges.

Within this context and the inborn defects of the RS image, we propose a novel interpolation algorithm based on rational function model. To start with, with the development of the RS image resolution, the data which should be transmitted between the earth and the satellite has increased dramatically. The rational function is speedy to calculate the unknown pixel and restore the full resolution of the RS image if we only store the low resolution RS image to accelerate the data transmission. Next, the spatial resolution, the observation width of the satellite-borne camera and the weight of the camera is three conflicting indices. For example, if the spatial resolution of the satellite-borne camera is doubled, the observation width of it decreased twice. Under the existing technical condition, the rational interpolation can alleviate this contradiction to a certain extent. In addition, since our algorithms give more consideration to the directional information, so the relatively good edge was preserved and the detail preservation was good enough. Finally, there is a free parameter in the rational spline function and the interpolation surface will be more adaptable to the characteristics of the human visual system and more suitable for human observation by adjusting this parameter, so as to bring convenience for visual interpretation.

The remainder of this paper is organized as follows: Firstly, the rational interpolation function model is described; Secondly, the interpolation algorithm considered in the study is presented; Lastly, the experimental results and conclusions are shown in the final part of this paper.

\section{The Rational Interpolation Function Model}

In recent years, some methods (Zhang Caiming et al. 2013; Kwok-Wai Hung et al. 2012) could be used to construct patch to create the high-resolution RS images. A bivariate rational interpolation with parameters based on the function values has been studied in (Zhang Yunfeng et al. 2012; Zhang Yunfeng et al. 2011; Duan Q et al. 2006; Duan Q et al. 2006).

The interpolation function model determines the quality of the RS image interpolation. Moreover, the rational interpolation function with parameters is more accurate than the function with no parameters. Furthermore, the flexibility of rational interpolation function model based on triangular 
domain is much greater than that of others.

Definition of the Rational Interpolation Function: Let $\left\{\left(x_{i}, y_{i}, f_{i}, d_{i}\right), i=1,2, \ldots, n\right\}$ be the given scattered data arranged in parallel lines, where $f_{i}=f\left(x_{i}, y_{i}\right)$, and $d_{i}=\frac{\partial\left(x_{i}, y_{i}\right)}{\partial x}$.

For a triangular domain $T_{1}=\Delta V_{1} V_{2} V_{3}$, with vertices $\left\{V_{i}=\left(x_{i}, y_{i}\right), i=1,2,3\right\}$ and $y_{1}=y_{2}$, let $\gamma_{11}=\angle V_{3} V_{1} V_{2}$ be the angle between lines $V_{3} V_{1}$ and $V_{1} V_{2}$, and $\gamma_{12}$ be the angle between line $V_{2} V_{3}$ and the extension line of $V_{1} V_{2}$ (see Fig.2).

Denoting $\boldsymbol{h}=\boldsymbol{x}_{2}-\boldsymbol{x}_{1}, \boldsymbol{l}=\boldsymbol{y}_{3}-\boldsymbol{y}_{\mathbf{1}}$. For any point $\mathrm{Q}$ in the line $\boldsymbol{V}_{1} \boldsymbol{V}_{2}$, let $\boldsymbol{\beta}=\angle \boldsymbol{V}_{3} Q \boldsymbol{V}_{2}$ be the angle between lines $V_{3} Q$ and $Q V_{2}$, thus, $V_{1} Q=x_{3}-x_{1}-l \cot \beta$, and for any point $V(x, y)$ in the line $V_{3} Q$, $\cot \beta=\frac{x-x_{3}}{y-y_{3}}$, let $\theta=\frac{x_{3}-x_{1}-l \cot \beta}{h}$, and $\eta=\frac{y-y_{1}}{l}$. A rational cubic function is defined over the interval over $\left[x_{1}, x_{2}\right]$ as Formula (1).

$$
p(x)=\frac{(1-\theta)^{3} \alpha f_{1}+\theta(1-\theta)^{2} V+\theta^{2}(1-\theta) W+\theta^{3} f_{2}}{(1-\theta) \alpha+\theta}
$$

where

$$
\begin{aligned}
& V=(2 \alpha+1) f_{1}+\alpha h d_{1}, \\
& W=(\alpha+2) f_{2}-h d_{2},
\end{aligned}
$$

In order to facilitate the application of the image interpolation, formula (1) can be rewritten as the following form

$$
\begin{aligned}
P_{T_{1}}(x, y)= & \omega_{1}(\theta, \eta) f_{1}+\omega_{2}(\theta, \eta) f_{2}+\omega_{3}(\theta, \eta) f_{3} \\
& +\omega_{4}(\theta, \eta) h d_{1}+\omega_{5}(\theta, \eta) h d_{2}
\end{aligned}
$$

where

$$
\begin{aligned}
& \omega_{1}(\theta, \eta)=\frac{(1-\eta)(1-\theta)^{2}(\theta+\alpha \theta+\alpha)}{(1-\theta) \alpha+\theta}, \\
& \omega_{2}(\theta, \eta)=\frac{(1-\eta) \theta^{2}(2-\theta+(1-\theta) \alpha)}{(1-\theta) \alpha+\theta}, \\
& \omega_{3}(\theta, \eta)=\eta \\
& \omega_{4}(\theta, \eta)=\frac{(1-\eta) \theta(1-\theta)^{2} \alpha}{(1-\theta) \alpha+\theta}, \\
& \omega_{5}(\theta, \eta)=\frac{(1-\eta) \theta^{2}(1-\theta)}{(1-\theta) \alpha+\theta}
\end{aligned}
$$

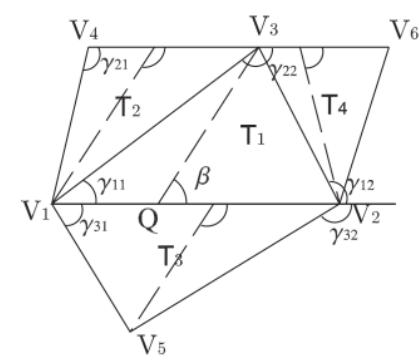

Fig.2 An Element of Subdivision

\section{The Interpolation Algorithm and Optimization}

In this section, we will introduce how to apply the rational function model to RS image interpolation. Fig. 3 shows the algorithm procedure. 


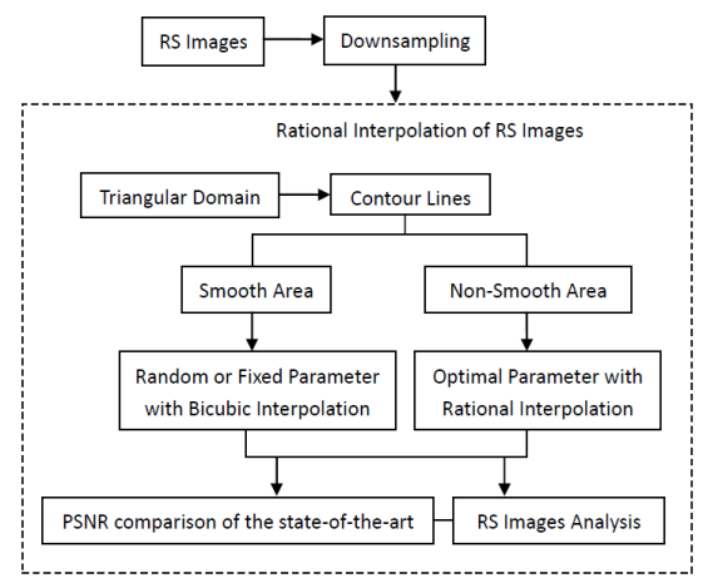

Fig.3 Flow Chart of the Proposed Algorithm

Transformation over Triangular Domain: As what mentioned before, the rational function is piecewise continuous. According to a certain rule, we transform every different triangular domain into the same domain by sorting the three vertices to improve the level of the rational function's continuity indirectly. Then we construct a more accuracy fitting surface locally by the combination of triangular patches and the effect of interpolation is enhanced. As shown in Fig.3, look at the two isosceles right triangles, the standard sequence of vertices are all labeled respectively. After rotating Fig. $3 \mathbf{1 8 0}^{\circ}$, we'll see the standard sequence of the right triangle. Other triangles' vertices are arranged by this rule. By this way, we finally turn the two different triangles definitional domain into the same one and the precision of interpolation was improved obviously.

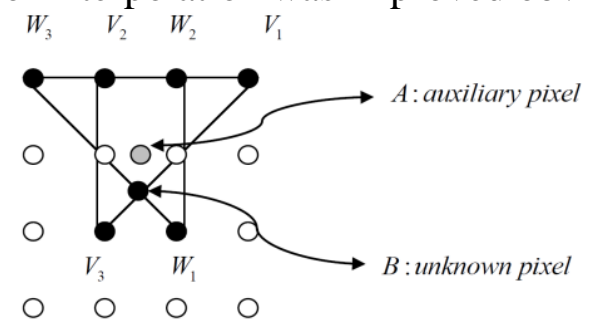

Fig.3 The Process of the Interpolation

As shown in Fig.3, we can obtain a value in the location of auxiliary pixel A in every triangle in the upper direction. Let's assume A_L stands for the corresponding value of left triangle and the other is A_R. Thus the auxiliary pixel A can be calculated by a weighted average of A_L and A_R. Then we use formula (3) to calculate the weight value of A_L and A_R. As shown in Fig.3, $f_{i}$ is arranged in orderly rows from left to right and up to bottom. Let $I_{L}=\left\{f_{1}, f_{2}, f_{3}, f_{6}, f_{7}, f_{11}\right\}$ and $I_{R}=\left\{f_{2}, f_{3}, f_{4}, f_{6}, f_{7}, f_{10}\right\}$ be two sets. Then calculating the two weight values by putting local variance $\operatorname{Var}\left(I_{L}\right)$ and $\operatorname{Var}\left(I_{R}\right)$ into formula (3) and we will get the value of auxiliary pixel A by the way mentioned above. The other three auxiliary pixels can be figured out in the same way.

$$
\begin{aligned}
& \omega_{1}=\frac{\operatorname{Var}\left(I_{R}\right)}{\operatorname{Var}\left(I_{L}\right)+\operatorname{Var}\left(I_{R}\right)} \\
& \omega_{2}=1-\omega_{1}
\end{aligned}
$$

In the case of the unknown pixel B, we got 4 accurate LR pixels and 4 auxiliary pixels. For the reason that the LR pixels are the accurate values so the weight value of them should be bigger than the value of the auxiliary pixels. Firstly, after lots of experiments, we assume the weight value of the $4 \mathrm{LR}$ pixels is $2 / 3$ and the weight value of the 4 auxiliary pixels is $1 / 3$ and $2 / 3$ can be the external weight value of the 4 LR pixels temporary. Secondly, considering the factor of the direction of the edge, we divide the $4 \mathrm{LR}$ pixels into two groups in the orthogonal direction and then calculating the local variance respectively and using formula (3) to figure out the internal weight value of each group. Finally, we will get the final weight value pixels by combining the external weight value with the internal weight value of the 4 LR pixels. Then the unknown pixel B can be calculated by a weighted average of 8 pixels ( 4 accurate LR pixels and 4 auxiliary pixels). As we mentioned earlier, if Fig. 3 was rotated by $45^{\circ}$ along the counter-clockwise direction and scaled by a 
factor of $2^{1 / 2}$, it becomes exactly the same as the step mentioned above. Therefore, the other three unknown pixels can be calculated in a similar way too.

Adaptive Decomposition of the Processed Image: According to the structure model of the rational interpolation function, we draw contour lines in every interpolation unit. Firstly, as shown in Fig.3, calculating the mean value $\bar{x}$ of the 16-connected neighbors of the processing region $\left(f_{i}\right.$ is arranged in orderly rows from left to right and up to bottom.). Secondly, figuring out the difference of LR pixel and $\bar{x}$, then we get four values $\Delta_{i}$ by using the following formula. As shown in Fig.4, if the four values have the same sign, the unknown pixel is in the smooth area. Otherwise, it is in the non-smooth area which has a characteristic of the tremendous change of the pixel values. In addition, we can also identify the pseudo direction of the edge by the sign we have already figured out above and the directional information is a key factor to influence the weight value of the unknown pixel too.

$$
\bar{x}=\frac{1}{16} \times \sum_{i=1}^{16} f_{i}, \quad \Lambda_{i}=\bar{x}-f_{j}, i=1,2,3,4, j=6,7,10,11
$$

For the RS image, the smooth area's structure is simple and the non-smooth area's structure is relatively complicated. In order to strike a balance between the speed and the quality of interpolation, we separate the RS image into different areas by drawing contour lines. For the smooth area, we interpolate the unknown pixel by using the traditional method, such as the Bi-cubic interpolation. Experience tells us these methods can handle the job pretty well in this area. For the non-smooth area, we interpolate the unknown pixel by using rational interpolation.
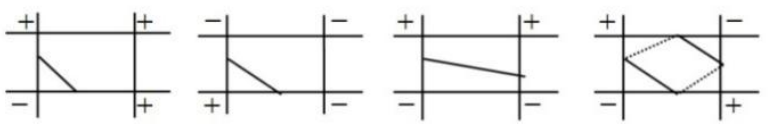

Fig.4 Boundary Detection Based on Contour Line non-smooth area, we will focus on how to calculate the optimal parameter. For the smooth area, after experiment we found that the parameter has little effect on the effect of interpolation so we just choose a random or fixed parameter in this area. The main objective of this section is to find out the optimal parameter in the non-smooth area.

Through the observation of Fig.3 and Fig.5 (Rotating the right triangle $180^{\circ}$ in Fig.3), we found that not only do we have three accurate pixels $V_{1}, V_{2}, V_{3}$ in every triangular domain but also we have three accurate pixels $V_{4}, V_{5}, V_{6}$, so we can deduce the three unknown parameters $\alpha_{1}, \alpha_{2}, \alpha_{3}$ by substitute $V_{4}, V_{5}, V_{6}$ into formula (2), then we calculate the average value of $\alpha_{1}, \alpha_{2}, \alpha_{3}$ and give it a symbol of $\bar{\alpha}$. At the end, we substitute $\bar{\alpha}$ and $V_{1}, V_{2}, V_{3}$ into formula (2) and we will get the value of the unknown pixel B by using the algorithm mentioned above.

\section{Experimental Results and Remarks}

Experiment Setting: Extensive experiments have been conducted to evaluate the proposed novel image rational interpolation technique in comparison with its predecessors. In order to prove the effectiveness of the algorithm proposed by this paper, we prepare to evaluate many aspects of the RS images, such as the image display quality after being magnified, the enhancement of the image edge or the texture area and so on. Five RS images are shown in Fig. 6 were used for testing the robustness and accuracy of the proposed method in different situations. The image sizes are all 512 $\times$ 512. The images were downsampled without low-pass filtering and upsampled two times. we decide to compare with the three other state-of-the-art methods: NEDI (Li Xin et al. 2001), DFDF (Zhang Lei et al. 2006), SAI ( Zhang Xiangjun et al. 2008) respectively. 


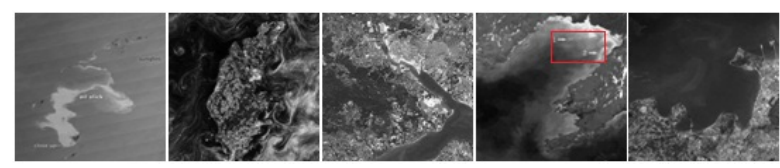

Fig.6 The benchmark images

To give a quantitative evaluation of the processed RS images, we use the widely-used peak signal-to-noise ratio (PSNR) as defined below:

$$
P S N R=20 * \log _{10}\left(\frac{255}{R M S E}\right)
$$

where

$$
R M S E=\sqrt{\frac{\sum_{i=0}^{M-1} \sum_{j=0}^{N-1}[O(i, j)-I(i, j)]^{2}}{M * N}}
$$

where $\mathrm{M}$ and $\mathrm{N}$ are the dimensions of the image. $O(i, j)$ is the original intensity at pixel $(i, j)$ and $I(i, j)$ is the interpolated intensity at $(i, j)$.

As shown in Table 1, it tabulates the PSNR results of the three different methods when applied to the five test images of Fig.6. On almost all instances, the proposed algorithm exceeds the PSNR values of the other methods. The average PSNR of our method is higher than that of the NEDI, DFDF, SAI, respectively. For the reason that it is hard to find out the difference between the processed RS images by different methods in IM 2, IM 3 and IM 5, so we just compare them with the PSNR value. Note that the proposed algorithm performs exceptionally well in IM_1, IM_2, IM_3, IM_4 which involves a good many of consistent geometric structures across resolutions, and performs relatively bad in IM_5 which involves serious misalignment problem of "pixel arrangement”. In almost all test RS images, the proposed method gives the highest PSNR and the best subjective display quality.

Table 1 The PSNR comparison of different methods

\begin{tabular}{|lllll|}
\hline & NEDI & DFDF & SAI & OURS \\
\hline IM_1 & 41.125 & 41.642 & 42.224 & 42.835 \\
IM_2 & 30.179 & 30.411 & 30.280 & 30.484 \\
IM_3 & 25.491 & 25.509 & 25.512 & 25.515 \\
IM_4 & 36.603 & 36.650 & 36.656 & 37.176 \\
IM_5 & 33.518 & 34.180 & 34.754 & 34.621 \\
Average & 33.383 & 33.678 & 33.885 & 34.126 \\
\hline
\end{tabular}

In this section, we will focus on the analysis of IM_1 and IM_4. As for IM_1, it is obvious that NEDI, DFDF, SAI and the proposed method produce some visually similar effects around edge area. However, the PSNR of the proposed method outperforms other methods. And if you look carefully enough, you'll find the edge preservation of the proposed method is relatively good than other methods. As for IM_4, In order to highlight the details of the RS image, we magnify the marked part with red line, as shown in Fig.11, NEDI, DFDF and SAI are blurred near the area pointed by the white arrow marked as 2 . The proposed method can perform well in the non-smooth area with a relatively sharp edge. So we can draw a conclusion that the proposed algorithm has advantages in detail preservation and visual quality, compared to the three state-of-the-art algorithms. These characteristics are helpful to analyze the important information of the RS image, such as the contaminated area, the accurate pollution boundary and so on.

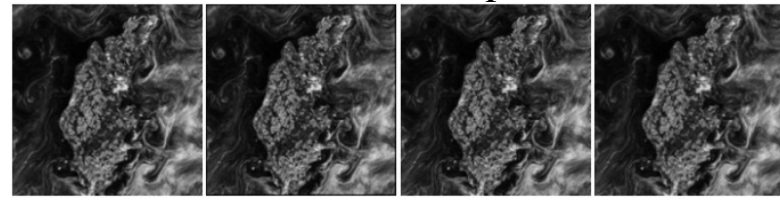

Fig.7 The comparison of IM_2

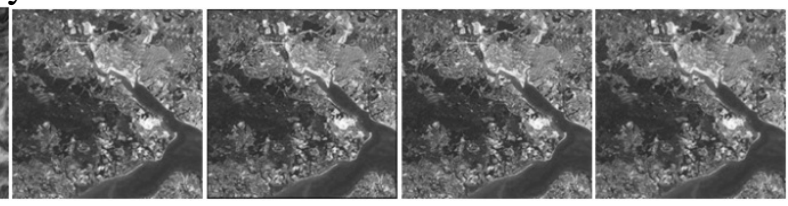

Fig.8 The comparison of IM_3 


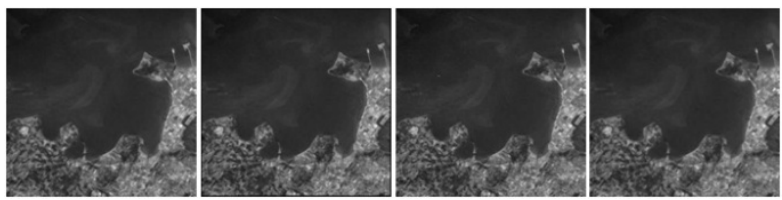

Fig.9 The comparison of IM_5
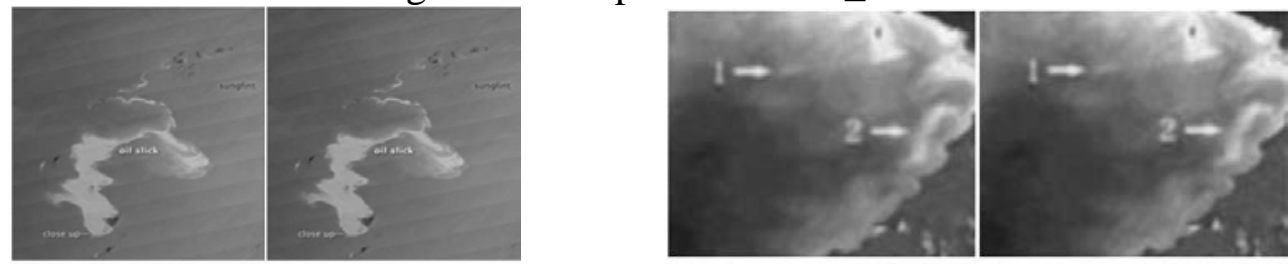

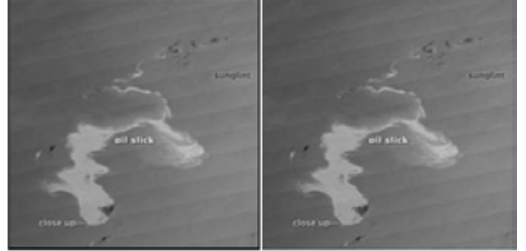

Fig.10 The comparison of IM_1

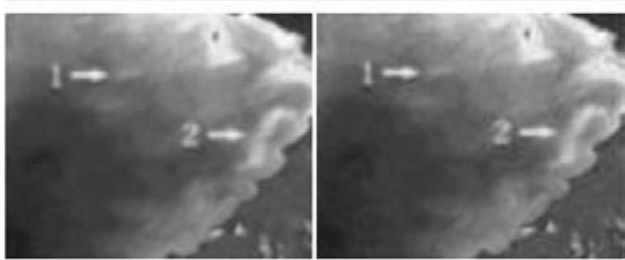

Fig.11 The comparison of IM_4

In summary, although the algorithms listed in Table 1 can improve spatial resolution of the RS image, but these types of methods sometimes generate speckle noise or distortion of edges. In order to solve this problem, the rational spline function is proposed. The great significance of the RS image rational interpolation is that we can't identify the critical aspects, such as the area of the pollutant, the tiny pollution sources and the pollution level, just with the low-resolution RS images for the reason that they are fuzzy and the texture or the edge is not shape enough. Because the primary method of analyzing the RS image is visual interpretation and the boundary of the pollutant is very hard to measure without processing the low-resolution images, it will be pretty hard to make the corresponding pollution management policy in the following work.

RS Image Analysis by EMPSR: For the analysis of the water quality of the RS imagery, pollutant coverage products from the Experimental Marine Pollution Surveillance Report (EMPSR) are used to validate the results. The EMPSR is an experimental product produced by the National Oceanic and Atmospheric Administration. Analysts interpret SAR and visible RS imagery from satellites to estimate the surface oil extent of the spill (NOAA/NESDIS).

While RS Image Analysis is not the main focus of the current paper, we give a brief introduction of this step just for the completion of the current work.

\section{Conclusions}

Conventional RS image enhancement methods generally make the enlarged images have blurred edges and annoying artifacts, the main reason is that they are not well adapt to the characteristic of RS images. In this paper, we present a novel rational interpolation algorithm and it produces the resized RS images with better quality. The new technique is superior to all the methods are listed in average PSNR measure and make the RS images more easily to be interpreted directly. This is of great significance for the water pollution monitoring and making the corresponding management strategies in the following work.

\section{Acknowledgement}

This work is supported by the National Nature Foundation of China (61373080, 61373078, 61303088, 61402261).

\section{References}

[1] R.G Key (1981) Cubic convolution interpolation for digital image processing. IEEE Trans Acoust, Speech and Signal and Processing 29:1153-1160 
[2] H.S.Hou, H.C Andrew (1978) Cubic splines for image interpolation and digital filtering. IEEE Trans Acoust, Speech and Signal and Processing 26:508-517

[3] Liu Xiaodi, Tu Guofang (2003) Wavelet Bi-linear interpolation in RS image. the Graduate school of the Chinese Academy of Sciences 20(1):39-43

[4] Liu Bo, Qu Youshan, Feng Guilan (2006) Maximal PSNR Wavelet Bi-linear Interpolation Iterative Algorithm in RS image. Acta Photonica Sinica 35(3):468-472

[5] Tu Guofang, Zhang Can, Wu Jiankang, Liu Xiaozhou (2005) RS image processing using wavelet fractal interpolation. In proceedings of International Conference on Communications, Circuits and systems Volume 2:701-706

[6] Qu Youshan, Tian Weijian, Li Yingcai, Zhang Wei, Da Xuanfu (2004) Improvement of the Space Resolution of the Optical RS image By the Wavelet Bi-cubic Interpolation. Acta Photonica Sinica 33(5) : 601-604

[7] Zhang can, Tu Guofang, Liu Xiaozhou (2005) RS image Processing Using Wavelet Fractial Interpolation. Journal of Computer Research and Development 42(2) : 247-251

[8] M. N. Do and M. Vetterli (2005) The contourlet transform: an efficient directional multiresolution image representation. IEEE Transactions on Image Processing 14(12): 2091-2106

[9] M. N. Do, M. Vetterli, Contourlets (2002) a directional multiresolution image representation. Proceedings of International Conference on Image Processing. Piscateway, USA: IEEE Press 2002: 357-360

[10] A. L. Cunha, J. Zhou, M. N. Do (2006) The Nonsubsampled Contourlet Transform: Theory, Design, and Applications. IEEE Trans. Image Processing 15(10): 3089-3101

[11] J. Zhou, A. L. Cunha, M. N. Do (2005) Nonsubsampled Contourlet Transform: construction and application in enhancement. Proceedings of International Conference on Image Processing. Genoa, Italy 2005

[12] Li X, Orchard M T (2001) New edge-directed interpolation. IEEE Transactions on Image Processing 10: 1521-1527

[13] Zhang L, Wu X (2006) Image interpolation via directional filtering and data fusion. IEEE Transactions on Image Processing 15: 2226-2238

[14] Zhang X, Wu X (2008) Image interpolation by adaptive 2-D autoregressive modeling and soft-decision estimation. IEEE Transactions on Image Processing 17: 887-896

[15] Zhang Caiming, Zhang Xin, Li Xuemei, Cheng Fuhua (2013) Cubic Surface Fitting To Image With Edges As Constraints. The international Conference on Image Processing (ICIP) 1046-1050

[16] Kwok-Wai Hung, Wan-Chi Siu (2012) Robust Soft-Decision Interpolation Using Weighted Least Squares. IEEE Transactions On Image Processing 21:1061-1069

[17] Zhang Yunfeng, Bao Fangxun, Zhang Caiming (2012) A weighted bivariate blending rational interpolation function and visualization control. Journal of Computational Analysis and Applications 14: 1303-1321

[18] Zhang Yunfeng, Bao Fangxun, Zhang Caiming (2011) Local Shape Control of a Bivariate Rational Interpolating Surface with Mixing Conditions. ISVD(2011): 200-205

[19] Duan Q, Zhang H L, Zhang Y F (2006) Bounded Property and Point Control of a Bivariate Rational Interpolating Surface. Computers and Mathematics with Applications 52: 975-984

[20] Duan Q, Zhang Y F, Twizell E H (2006) A bivariate rational interpolation and the properties. Applied Mathematics and Computation 179: 190-199

[21] T. J. Crone, M. Tolstoy (2010) Magnitude of the 2010 Gulf of Mexico oil leak. Science 
[22] Gregoire Mercier, Fanny Girard-Ardhuin (2006) Partially Supervised Oil-Slick Detection by SAR Imagery Using Kernel Expansion. IEEE Transactions on Geoscience and Remote Sensing 44:2839-2846

[23] Maurizio Migliaccio, Attilio Gambardella, Ferdinando Numziata, Masanobu Shimada, Osamu Isoguchi (2009) The PALSAR Polarimetric Mode for Sea Oil Slick Observation. IEEE Transactions on Geoscience and Remote Sensing 47:4032-4041

[24]Nunziata $\quad$ F, $\quad$ Sobieski $\quad$ P, $\quad$ Migliaccio $\quad M \quad$ (2009) The Two-Scale BPM Scattering Model for Sea BiogenicSlicks Contrast. IEEE Transactions on Geoscience and Remote Sensing 47:1949-1956

[25] Richard D.Lindsley, David G.Long (2012) Mapping Surface Oil Extent From the Deepwater Horizon Oil Spill Using ASCAT Backscatter. IEEE Transactions on Geoscience and Remote Sensing 50:2534-2541

[26] Stine Skrunes, Camilla Brekke, Torbjorn Eloft, Vladimir Kudryavtsev (2015) Comparing Near-Coincident C- and X-Band SAR Acquisitions of Marine Oil Spills. IEEE Transactions on Geoscience and Remote Sensing 53:1958-1975

[27] NOAA/NESDIS, Satellite Derived Surface Oil Analysis Products-Deepwater Horizon. [Online]. Available: http://www.ssd.noaa.gov/PS/MPS/deepwater.html 